Results 92 feedback forms were returned (20 [22\%] from the hub and 72 [78\%] from four spokes). 4 (5\%) males: 86 (96\%) females, median age 30 years (range 16-64). Knowledge of services offered improved from median 4/10 (range 1-10/10) to median 10/10 (range 1-10/10) after reading the leaflet. 33/66 $(50 \%)$ patients not originally attending for an STI screen would consider or agree to screening after reading the leaflet $(36 / 82$ [44\%] for HIV testing respectively). The leaflet received an overall rating of median 10/10 (range 5-10/10).

Discussion/conclusion Overall the leaflet was well received and improved patient's knowledge of services offered, and uptake of STI/HIV testing. Females provided the majority of feedback most likely due to spokes previously providing primarily contraceptive services. More work needs to be done to encourage males to attend the spoke clinics.

\section{P170 ASSESS THE RISK BEHAVIOURS AND SAFER SEX PRACTICES AMONG MALE ATTENDEES IN A SEXUAL HEALTH SETTING}

Dilmini Mendis*, Lilani Rajapaksa, PSK Gunathunga, M Jayalath, RC Fernando. National STD/AIDS Control Programe, Colombo, UK

\subsection{6/sextrans-2015-052126.213}

Background/introduction During the year 2011, 8511 males received services from the sexual health clinics island wide. At present there is only limited information on the risk behaviours of male attendees. Information on risk behaviours related to STI /HIV transmission is helpful in planning suitable prevention interventions.

Aim(s)/objectives The objectives were to determine the sexual partners responsible for transmitting STI/HIV and to understand the practice of safer sex.

Methods Study was a clinic based prospective study conducted for a one year period using an interviewer administered questionnaire.

Results 983 attendees were interviewed. 50\% admitted sex with a casual female, $12 \%$ with a casual male, and $13 \%$ with CSW (commercial sex workers). $20.5 \%$ used alcohol frequently and $5.9 \%$ used drugs and $1.4 \%$ injected. $6.7 \%$ gonorrhoea, $8.2 \%$ nonspecific urethritis (NSU), 7.5\% herpes and $0.7 \%$ HIV were transmitted by CSWs. Female casual partners were responsible for $3.7 \%$ gonorrhoea, $8.3 \%$ NSU, $6.6 \%$ herpes and $0.8 \%$ HIV. MSM contacts were responsible for $10.6 \%$ of gonorrhoea, $4.5 \%$ NSU, 7.6\% of infectious syphilis and $0.8 \%$ of HIV. Only 9\% used condoms correctly. Non use of condoms were not due to unavailability but for other reasons as worried about satisfaction $(24.6 \%)$ and faith in the partner (25.6\%).

Discussion/conclusion Casual partners for unsafe sex is a concern. MSM and CSW are remained as an important source of infection. More males contracted infections via casual partners. Low condom use remains another concern. Therefore strategies used for prevention need to be revisited also emphasising on general population where casual partners represent.

\section{P171 ACTIVE RECALL OF HIGH-RISK MSM BY TEXT MESSAGE}

Gary Whitlock, Oscar Duke, Nneka Nwokolo*, Alan McOwan. Chelsea \& Westminster Hospital, 56 Dean Street, London, UK

10.1136/sextrans-2015-052126.214
Background/introduction PHE recommends high risk MSM test 3 monthly. We introduced recall of high-risk MSM for HIV/sexually transmitted infection (STI) testing by short message service (SMS).

Aim(s)/objectives To assess effectiveness of SMS recall by rescreening rate and number of incident STIs.

Methods From January 2014, MSM who reported condomless anal intercourse with a non-regular partner in the last 3 months were offered an SMS 3 months later inviting them to rescreen. We compared the testing rate of the first 100 eligible MSM in the 12 weeks following SMS with a historical control group of 100 MSM who attended in January 2013. Proportions were compared using a two-tailed Z-test.

Results Median age was 30 y (IQR: 26-36 y) for SMS group and 29y (IQR: $25-35$ y) in controls. $44 \%$ of SMS group retested compared with $19 \%$ of controls ( $p<0.001$ ). 32\% of SMS group were diagnosed with an STI at retest (14/44; SMS) vs. $16 \%(3 / 19$; control). HIV was diagnosed in 2 of SMS group and 1 in control group at retest.

Discussion/conclusion Active SMS recall for MSM is associated with a statistically significantly higher retesting rate. The high proportion of MSM with STIs at re-screening reinforces the importance of active recall, especially using SMS reminders which are cheap and easy to facilitate.

\section{P172 IMPLEMENTATION OF ALCOHOL SCREENING IN PATIENTS ATTENDING A LARGE WALK-IN SEXUAL HEALTH SERVICE WITHIN LONDON}

Chantal Oxenham*, Alexander Campbell Guild, Salima Watfa, Olamide Dosekun, Ramona Malek, Lydia Hodson, Dawn Wilkinson. Imperial College Healthcare NHS Trust, London, UK

\subsection{6/sextrans-2015-052126.215}

Background UK national guidelines advocate a role for sexual health services to offer routine screening for high-risk alcohol consumption in patients. Screening for alcohol misuse and offering brief interventions in this setting has been shown to be acceptable to clinicians and patients. In August 2014 the Fast Alcohol Screening Test (FAST) was incorporated into the sexual history proforma in a London Genitourinary Medicine (GUM) clinic.

Aim An audit was undertaken to assess the use of the FAST tool and management of patients with a positive FAST result.

Methods A retrospective case-notes review of randomly selected patients attending the GUM clinic in October 2014 was performed. Information was collected on patient demographics, sexual history, sexually transmitted infections, completion of FAST tool and action dependent on outcome of risk assessment.

Results 169 case notes were reviewed: 55\% female and 45\% male, mean age was 30 (range 17-74) years. The FAST tool was completed in $87 \%(147 / 169)$ of case notes. Of patients screened, $86 \%$ (127) identified as low risk, $10 \%$ (15) increasing and $4 \%$ (5) high risk (hazardous drinkers). Of hazardous drinkers, 90\% (18) had a documented action for risk reduction; 56\% (10) had verbal advice documented, 22\% (4) accepted written advice, $22 \%$ (4) accepted referral to a sexual health advisor.

Conclusion Clinician completion of the FAST tool within the sexual history proforma in a busy clinic was high, with some scope for improvement. Of the relatively low number of hazardous drinkers identified, most accepted only brief verbal advice in clinic. 